\title{
What's in a name? Issues to consider when naming Mendelian disorders
}

\author{
Sonja A. Rasmussen, MD, MS ${ }^{1}{ }^{1}$ and Ada Hamosh, MD, $\mathrm{MPH}^{2}$ \\ for the OMIM curators \\ Joanna Amberger ${ }^{2}$, Cassandra Arnold ${ }^{2}$, Carol Bocchini ${ }^{2}$, Marla J. F. O’Neill ${ }^{2}$ and Anne Stumpf ${ }^{2}$
}

Genetics in Medicine (2020) 22:1573-1575; https://doi.org/10.1038/s41436-020-0851-0

Extraordinary advances in the field of genetics ${ }^{1}$ have led to the identification of hundreds of new Mendelian disorders (defined here as disorders caused by pathogenic variants in a single gene) and the genes that cause them. In the last ten years, over 2500 new single-gene conditions were added to OMIM (https://omim.org/), the National Institutes of Health (NIH)-funded catalog of inherited phenotypes, genes, and the relationships between them. Identification of new phenotypes and their associated genes has transformed the care of persons with Mendelian disorders through improved diagnostic testing, recurrence risk estimation, disease management, and in some cases, treatment. However, the huge number of new phenotypes has resulted in a challenge to the field-what to name these new conditions.

Disease naming is central to medicine. A common terminology is necessary for the gathering and exchange of information among health-care providers, researchers, and patients and their families. For centuries, disease naming has been controversial, as illustrated by the more than 400 names for syphilis since the 15 th century ${ }^{2}$ to the present day controversy regarding the name of coronavirus disease 2019 (COVID-19) caused by infection with the SARS-CoV-2 virus. ${ }^{3}$ Victor McKusick began the central task of naming genetic diseases in the 1960s when he created Mendelian Inheritance in Man, the precursor to the current OMIM catalog. ${ }^{4}$ Even before the recent proliferation of new phenotypes, McKusick recognized challenges to naming Mendelian disorders, noting that naming a phenotype shortly after its recognition is particularly challenging because key features might change as more patients are described. Thus Mendelian Inheritance in Man was designed to provide each entry with a unique MIM number that remains stable, allowing for subsequent changes in phenotype names if needed, with the previous names being listed in the entry to ease searching and limit confusion in the field.
The recent onslaught of new phenotypes and genes has prompted some to propose a new approach to phenotype naming: naming a phenotype after the symbol for the causative gene. Gene names are established through the HUGO Gene Nomenclature Committee, ${ }^{5}$ which assigns unique symbols and names to human genes, based on function, sequence similarity, homology in another species, clone designation, or chromosome location. Some of the newly identified phenotypes have been recognized using "reverse phenotyping," in which a pathogenic gene variant is identified in a group of patients first, followed by identification and description of the associated phenotype: identification of a gene before describing the phenotype might make naming phenotypes after genes appear to be a logical approach. Some examples of the approach of giving phenotypes names of causative genes include DICER1 syndrome, ${ }^{7}$ a condition that increases the risk of certain malignant and benign tumors (MIM 138800, MIM 601200 , and MIM 180295), caused by pathogenic variants in the DICER1 gene (MIM 606241); FOXG1 syndrome, ${ }^{8}$ a congenital variant of Rett syndrome (MIM 613454), caused by pathogenic variants in the FOXG1 gene (MIM 164874); and MYH9-related disorders, ${ }^{9}$ characterized by macrothrombocytopenia and granulocyte inclusions, with or without nephritis or sensorineural hearing loss (MIM 155100), caused by pathogenic variants in the MYH9 gene (MIM 160775). However, we believe that naming phenotypes after genes leads to confusion among patients and their health-care providers and could unnecessarily segregate Mendelian disorders from the rest of the medical field. This could lead to even further confusion if a gene symbol changes, which sometimes happens as more is understood about a gene's function. Furthermore, it obfuscates text mining and machine learning.

Ideally, names should be medically informative; meaningful; accessible across disciplines, including those distinct from the

\footnotetext{
${ }^{1}$ Departments of Pediatrics and Epidemiology, University of Florida College of Medicine and College of Public Health and Health Professions, Gainesville, FL, USA; ${ }^{2}$ Department of Genetic Medicine, Johns Hopkins University, Baltimore, MD, USA. Correspondence: Ada Hamosh (ahamosh@jhmi.edu)
} 
field of genetics; and amenable to administrative coding for billing purposes. Patients come to medical attention because they have clinical manifestations requiring management and treatment. Using a clinically based name to refer to a phenotype is especially helpful in guiding patient care for people who likely have a genetic disease, but have not had genetic testing performed. Similarly, naming genetic disorders after genes may place inappropriate focus on the genetic alteration rather than on the patient. Patients with genetic disease receive care in many subspecialties of medicine; naming genetic disorders after genes might separate or isolate these disorders from other medical disorders and from practitioners who are not directly involved in genetic medicine. Naming disorders after genes also discounts that certain Mendelian disorders should be considered together, even though they are caused by different genes, because treatment regimens and prognoses are similar. An important goal of naming diseases is to assist clinicians in making a diagnosis so treatment and prognosis can be provided; naming disorders after genes might make this connection more obscure.

Other flaws in the logic of naming genetic disorders after genes are related to phenotypic diversity and genetic heterogeneity. Phenotypic diversity refers to many clinically distinct phenotypes associated with variants in a single gene. More than one-third of known disease genes cause more than one phenotype. This phenomenon is illustrated by heterozygous pathogenic variants in the FBN1 gene (MIM 134797) (Supplemental Table) that have been associated with eight distinct phenotypes, ranging from isolated ectopia lentis (MIM 129600) to Weill-Marchesani syndrome (MIM 608328), characterized by short stature, brachydactyly, joint stiffness, and eye anomalies, to Marfan syndrome (MIM 154700), characterized by tall stature and risk for aortic aneurysm. Naming conditions after a causative gene that produces several different phenotypes could be misleading not only to affected patients and their families, but also to their health-care providers. As an example, a patient with a newly diagnosed FBN1 disorder or a clinician caring for him or her might anticipate an increased risk for aortic dissection, even though the patient might only be at risk for lens dislocation.

Genetic heterogeneity, which refers to more than one gene causing a particular phenotype, is also common and would complicate naming genetic disorders after genes. For example, Weill-Marchesani syndrome is caused by pathogenic variants in any of four different genes and is inherited in an autosomal dominant or autosomal recessive manner depending on the gene. While it may be critical to know the specific genetic defect for a particular phenotype, it is equally important to group similar phenotypes together in a phenotypic series. Phenotypic series, created when several phenotype entries are similar in their clinical manifestations, are generated to allow viewing of the genetic heterogeneity of phenotypes. Another example, long QT syndrome, may be caused by pathogenic variants in 15 different genes, but it is still essential for patients to be seen in a clinic with expertise in cardiac rhythm disorders to ensure proper diagnosis and treatment.
Finally, gene names are often cumbersome and less meaningful, making them difficult to remember. For example, one type of retinitis pigmentosa (retinitis pigmentosa 10) (MIM 180105) is caused by pathogenic variants in the IMPDH1 (or inosine-5-prime-monophosphate dehydrogenase, type I) gene (MIM 146690). This name provides little information to patients, their families, or their health-care providers and would be challenging to remember. One could argue that remembering which of the 88 types of retinitis pigmentosa the patient has (named retinitis pigmentosa 1retinitis pigmentosa-88 in our scheme) would also be difficult. However, including the name of the phenotype allows for health-care providers to recognize the disorder as a progressive eye disease.

Mendelian phenotypes of course are much more complicated than the single genes that cause them. As noted by Loscalzo and colleagues, ${ }^{10}$ how a disease phenotype presents reflects the results of a genetic network that includes the causative gene as well as disease-modifying genes all in the context of a changing environmental framework. Although this approach is helpful when considering the phenotype seen in an individual, it does not eliminate the need for a name to allow health-care providers, researchers, and patients to describe a particular disorder.

We propose that phenotype naming in genetics continue to follow the guidelines set by McKusick in the 12th edition of Mendelian Inheritance in Man. Phenotype names should be unique, enhance clinical care and classification, and be easy to communicate. If a phenotype name exists in OMIM, the existing name should be kept to avoid confusion. If the phenotype is similar to that of an existing phenotype, a phenotypic series can be developed, with the phenotype receiving the same name, but numbered sequentially. If a phenotype is new, the three to five most clinically significant features can be selected and used in the name or used to create an acronym or initialism that is both informative and memorable. If features are too numerous or variable, eponymous names, using the names of the authors who first described or established the phenotype-gene connection, can be used, which connects eponymous phenotypes to the papers that described them. Gene and phenotype names should develop independently according to the growing knowledge of the function of the gene and of the characteristic features of the phenotype, respectively. This approach to phenotype naming will provide the greatest benefit to health-care providers, researchers, and most importantly, to patients and their families.

\section{SUPPLEMENTARY INFORMATION}

The online version of this article (https://doi.org/10.1038/s41436020-0851-0) contains supplementary material, which is available to authorized users.

\section{FUNDING}

OMIM is funded by the National Human Genome Research Institute: U41HG006627. 


\section{DISCLOSURE}

The authors declare no conflicts of interest.

Publisher's note Springer Nature remains neutral with regard to jurisdictional claims in published maps and institutional affiliations.

\section{REFERENCES}

1. Adams DR, Eng CM. Next-generation sequencing to diagnose suspected genetic disorders. N Engl J Med. 2018;379:1353-1362.

2. Piro $A$, Distante $A E$, Tagarelli $A$. On allusive names for the syphilitic patient from the 16th to the 19th century: the role of dermatopathology. Am J Dermatopathol. 2017:39:949-950.

3. Joseph A. Disease caused by the novel coronavirus officially has a name: COVID-19. STAT. 11 February 2020. https://www.scientificamerican. com/article/disease-caused-by-the-novel-coronavirus-officially-has-aname-covid-19. Accessed 20 April 2020.

4. McKusick VA. Mendelian Inheritance in Man and its online version, OMIM. Am J Hum Genet. 2007;80:588-604.

5. Braschi B, Denny P, Gray K, et al. Genenames.org: the HGNC and VGNC resources in 2019. Nucleic Acids Res. 2019;47:D786-D792.

6. Jansen $S$, van der Werf IM, Innes AM, et al. De novo variants in FBXO11 cause a syndromic form of intellectual disability with behavioral problems and dysmorphisms. Eur J Hum Genet. 2019;27:738-746.

7. Apellaniz-Ruiz $M$, Segni $M$, Kettwig $M$, et al. Mesenchymal hamartoma of the liver and DICER1 syndrome. N Engl J Med. 2019;380:1834-1842.
8. Mitter D, Pringsheim $M$, Kaulisch $M$, et al. FOXG1 syndrome: genotypephenotype association in 83 patients with FOXG1 variants. Genet Med. 2018;20:98-108.

9. Bury L, Megy K, Stephens JC, et al. Next-generation sequencing for the diagnosis of MYH9-RD: predicting pathogenic variants. Hum Mutat. 2020;41:277-290.

10. Loscalzo J, Kohane I, Barabasi AL. Human disease classification in the postgenomic era: a complex systems approach to human pathobiology. Mol Syst Biol. 2007;3:124.

Open Access This article is licensed under a Creative Commons Attribution-NonCommercial-NoDerivatives 4.0 International License, which permits any non-commercial use, sharing, distribution and reproduction in any medium or format, as long as you give appropriate credit to the original author(s) and the source, and provide a link to the Creative Commons license. You do not have permission under this license to share adapted material derived from this article or parts of it. The images or other third party material in this article are included in the article's Creative Commons license, unless indicated otherwise in a credit line to the material. If material is not included in the article's Creative Commons license and your intended use is not permitted by statutory regulation or exceeds the permitted use, you will need to obtain permission directly from the copyright holder. To view a copy of this license, visit http://creativecommons.org/licenses/by-nc-nd/4.0/.

(c) The Author(s) 2020 